\title{
Anemia in CLL: Autoimmune Myelofibrosis with Literature Review
}

Yasir Khan MD1, Monica El-Masry ${ }^{1}$, Sherif Rezk MD², Susan O'Brien MD ${ }^{1,3}$

, Division of Hematology-Oncology ${ }^{1}$, Department of Pathology and Laboratory Medicine ${ }^{2}$ and Chao Family Comprehensive Cancer Center ${ }^{3}$, University of California Irvine, Orange, CA, USA

\section{Background:}

Autoimmune Myelofibrosis is a rare entity that occurs in the setting of autoimmune disorders, predominantly with rheumatologic diseases and rarely with malignancies. It is a clinical pathologic entity that was first described in 1994 in a patient with Systemic Lupus Erythematous, but since then it has been reported with lymphoid neoplasms, metastatic solid tumors, pulmonary hypertension, chronic infections and therapeutic agents (GMCSF). It is critical to differentiate Autoimmune myelofibrosis from Primary myelofibrosis.

\section{Case Presentation:}

25F (G2P2), developed cervical lymphadenopathy in Fall of 2014 in the setting of upper respiratory viral illness. Lymphadenopathy persisted; consequently, patient had biopsy and was diagnosed with Small Lymphocytic Leukemia. Subsequently, patient was treated with low dose radiation in early January 2016. A month later, patient presented with symptomatic anemia, with bone marrow biopsy remarkable for fibrosis and erythroid hypoplasia.

Diagnostic Workup:

WBC: 6.8; Hgb: 5.5, HCT: 16.1; PLT: 318, ALC: 3.264

Reticulocyte Production Index: 0; PT/INR: 12.7/1.0

Haptoglobin: 242; LDH: 498; Ferritin: 962;

Direct Antiglobulin Test: Negative IgG, Complement TSH: 0.44; EBV, CMV, Parvo, HIV, Hepatitis: Neg.

MDS/CLL/MPN: FISH Panel Negative; 46XX

Negative JAK2V167F, CALR exon 9

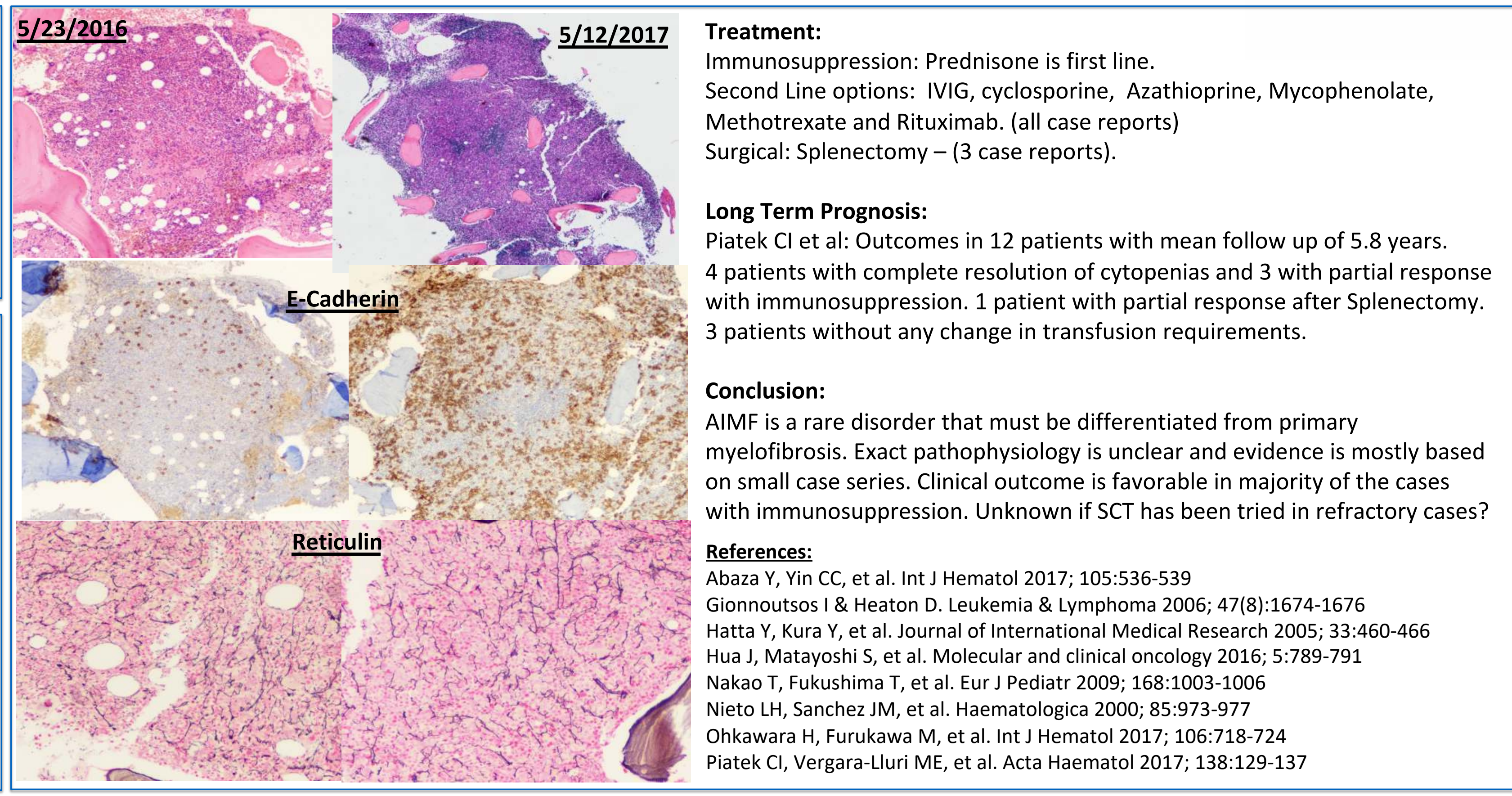

\section{Literature Review: (Case Series Based)}

\section{Diagnostic Criteria:}

1-Bone marrow fibrosis, MF-1-2/3

2-Lack of clustered or atypical megakaryocytes

3-Lack of myeloid or erythroid dysplasia, eosinophilia and basophilia

4-Lymphocyte infiltration of Bone Marrow

5 -Lack of osteosclerosis

6-Absent or mild Splenomegaly

7-Presence of Autoantibodies

8-Absence of clonal markers

\section{Pathophysiology:}

Exact pathophysiology is unclear. Disease is often associated with underlying autoimmune condition with immune dysregulation and T-cell dysfunction that leads to aberrant fibrogenic cytokine production. Harrison et al. illustrated high levels of fibrogenic cytokines in patients with AIMF compared to healthy controls that decreased with immunosuppressive agents. Role of spleen?

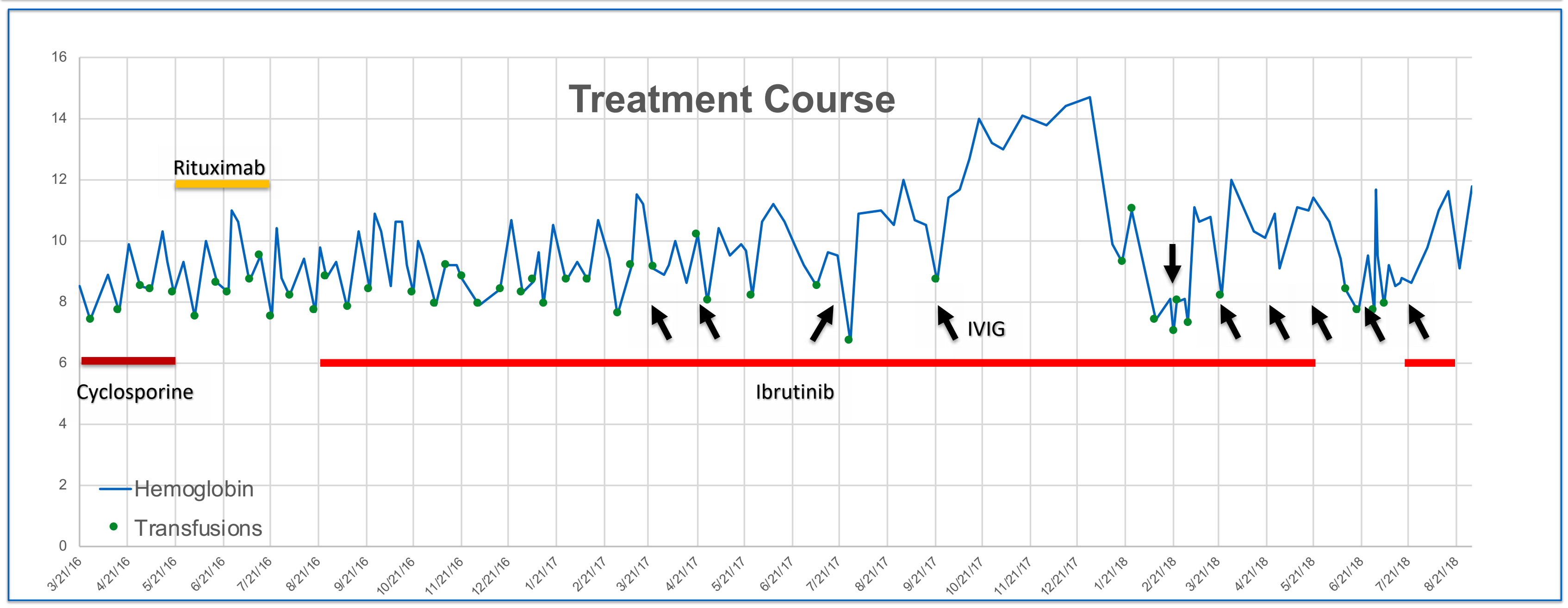

